\section{Biliary tract tuberculosis: An enigma}

\section{Introduction}

Mycobacterium tuberculosis infection of biliary tract as an isolated presentation is a rare entity even in endemic areas. A literature survey till date shows less than 120 cases of gall bladder tuberculosis (GBTB) ${ }^{1}$ and nearly about 18 cases of tuberculous biliary stricture (TBS) ${ }^{2}$ having been reported. Majority of the reported cases have been diagnosed only postoperatively due to lack of specific clinical or radiological pathognomonic features for biliary tract TB (BTB). Here we report 3 cases of BTB; case 1- GBTB with TBS, case 2- TBS, case 3- GBTB.

\section{Case Reports}

\section{Case 1}

A 48 year old male presented with jaundice, dyspepsia and significant loss of weight for 6 months. There was no fever, melena, pruritis or passage of clay coloured stools or past history of tuberculosis. He was diagnosed to be HBsAg positive 4 years back. He had fluctuating direct hyperbilirubinemia with total bilirubin levels ranging from $4-10 \mathrm{mg} / \mathrm{dl}$ and was on native treatment.

When he presented to us, MRCP showed an abrupt cut off of the mid CBD (Figure 1) and his chest X-ray was normal. CA 19-9 - 31.2 Units/ml. HIV 1 \& 2 ELISA was non reactive. ERCP revealed a tight mid CBD stricture and was non-stentable. ERCP brush cytology was not contributory. A diagnosis of possible malignant mid CBD stricture was made.

Peroperatively there was a mass in the neck of the gall bladder extending into the adjacent CBD causing a stricture with few enlarged pericholedochal nodes and interaortocaval lymph node. There were no stones in the gall bladder. A frozen section biopsy of the interaortocaval lymph node revealed acaseating granulomatous lesion. An open cholecystectomy with Roux en Y hepaticojejunostomy was done. HPE revealed tuberculous lesion in the neck of the gall bladder with no evidence of malignancy (Figure 2). WHO category I antituberculosis therapy was started and the patient is recuperating now.

\section{Case 2}

A 56 year old male had history of painless progressive jaundice for 2 months duration. On evaluation there

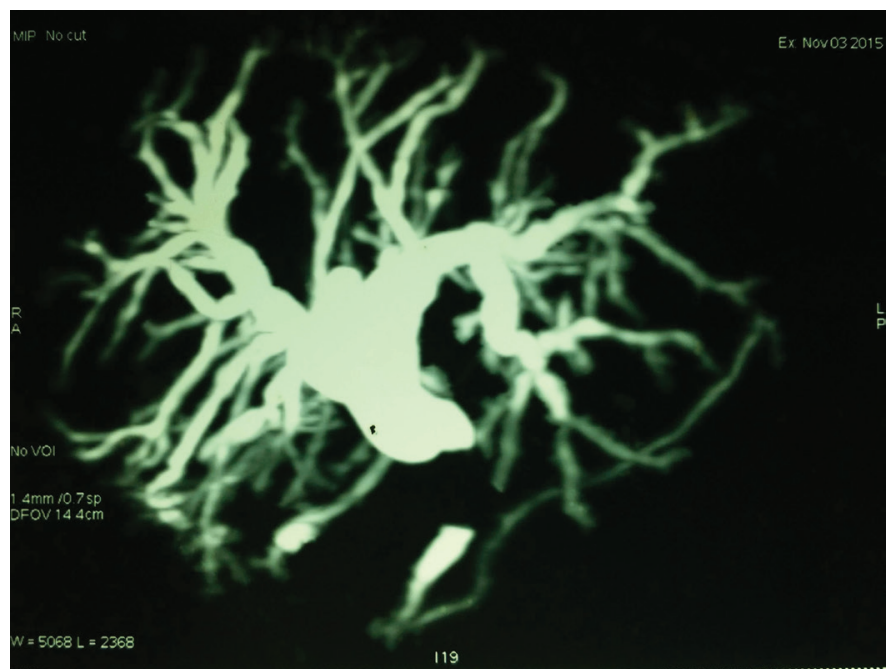

Figure 1: MRCP showing mid CBD stricture.

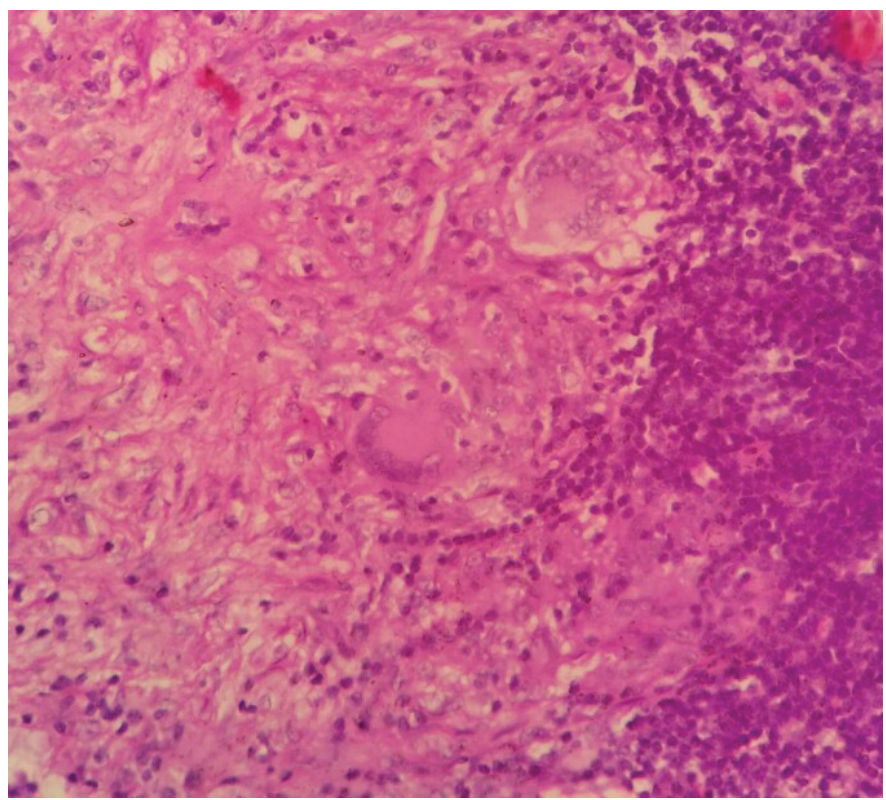

Figure 2: High power view (40x) of inter aortocaval lymphnode showing epitheliod cells with Langhan's giant cell. 
was a mid CBD stricture. ERCP and brush cytology was positive for malignant cells. He underwent a radical excision of extrahepatic bile duct and a Roux en Y hepaticojejunostomy. Post operatively the HPE showed tuberculosis of the common bile duct with no evidence of malignancy. The brush cytology was reviewed and a final diagnosis of BTB was confirmed. He had completed a course of antituberculosis treatment but lost follow up after that.

Six years later he presented to us with cholangitis. A stricture at the hepaticojejunostomy site was detected on MRCP and hence he underwent percutaneous transhepaticballoon dilatation of the stricture with PTBD (Figure 3) and is responding well now. This case entails the importance of long term follow up of patients with BTB as they are likely to develop late strictures.

\section{Case 3}

A 50 year old male underwent open subtotal cholecystectomy for acute calculous cholecystitis. He developed a persistent sinus in the surgical scar few months later, a biopsy of which revealed a tuberculous granuloma. He had persistent upper abdominal discomfort. A careful retrospective review of the removed gall bladder specimen revealed a tuberculous focus (Figure 4).

\section{Discussion}

Biliary tract tuberculosis is rare because of the alkalinity of bile and the inhibitory effect of bile salts in bile. ${ }^{3}$ Cholelithiasis, cystic duct obstruction with an alteration in the composition of bileis important for the development of GBTB. In $70 \%$ of GBTB, cholelithiasis is present and it is common in females. ${ }^{4}$ But in our series all were male patients. GBTB can present as acute or chronic cholecystitis or may mimic gall bladder cancer. BTB has protean manifestations and hence isolated BTB is difficult to diagnose unless a high index of clinical suspicion exists. BTB must be suspected irrespective of the age and gender when the clinical presentation is atypical and in immunocompromised individuals. TBS must be suspected when a patient has fluctuating jaundice. When suspected, an ERCP with bile sampling and a Ziehl

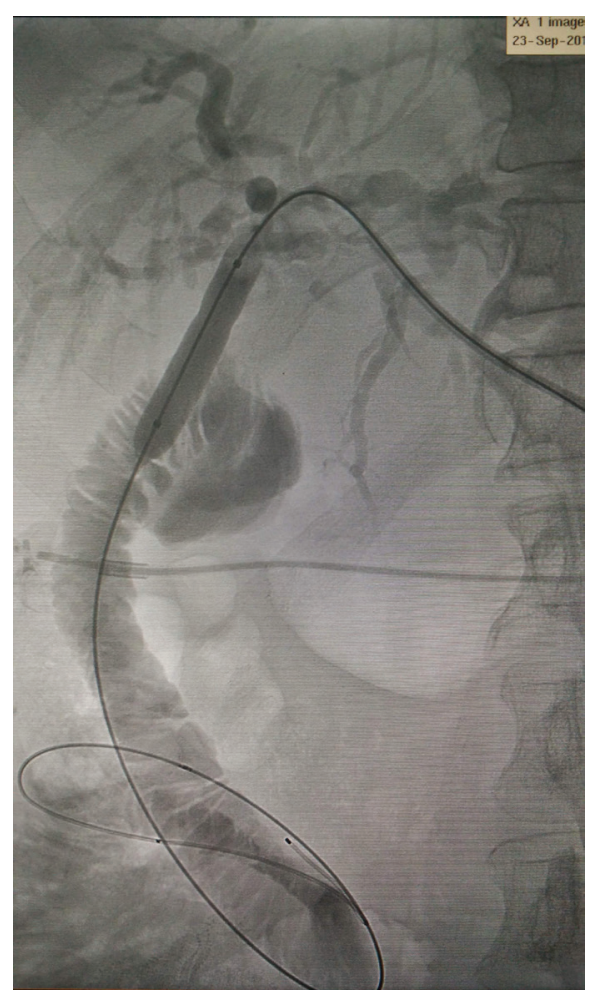

Figure 3: Percutaneous transhepatic balloon dilatation of hepatico jejunostomy stricture being done.

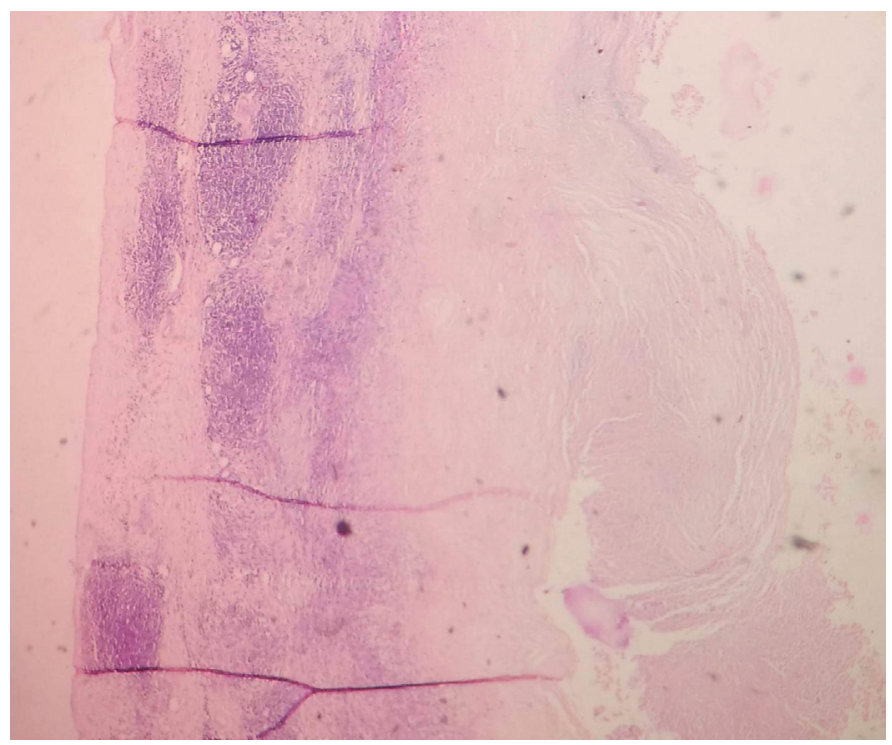

Figure 4: Low power view (10x) showing gall bladder wall with epitheloid granulomas with Langhan's giant cell.

Neelsen stain must be done. Multiplex PCR of bileor tissue samples is a more sensitive and specific method to detect the bacilli. ${ }^{5}$ Endoscopic ultrasound guided biopsy 
of suspicious lesions or adjacent lymph nodes may help in prompt diagnosis. BTB usually masquerades as cholangiocarcinoma and a prompt diagnosis helps to avoid major surgery and its ensuing morbidity. When in doubt a diagnostic laparoscopy with biopsy of suspicious lesions can be attempted. Frozen section biopsy prior to resorting to major surgical resections is a useful armamentarium when BTB is suspected. All surgically removed gall bladders must be carefully inspected followed by histopathological examination of suspicious foci. Post operative persistence of symptoms must not be branded as Post Cholecystectomy Syndrome and BTB must be considered in the appropriate setting. Treatment of BTB is medical therapy along with interventional endoscopic procedures to relieve biliary obstruction. Patients treated for TBS should be followed up long term for timely detection and prompt treatment of late strictures prior to the development of secondary biliary cirrhosis.

\section{SOMASEKAR DURAIRAJAN ANAND LAKSHMANAN ABDUL REHMAN ABDUL JAMEEL DURAIRAJ SEGAMALAI AMUDHAN ANBALAGAN KANNAN DEVY GOUNDER \\ Institute of Surgical Gastroenterology, Madras Medical College, Chennai-600003. \\ Correspondence: Prof. D. Kannan Email:_malarkan08@gmail.com}

\section{References}

1. Xu X-F, Yu R-S, Qiu L-L,et al. Gallbladder Tuberculosis: CT Findings with Histopathologic Correlation. Korean Journal of Radiology. 2011;12(2):196-202.

2. Padhiari RK, Ramesh M., G.P P, Ahmed N. Tuberculosis Biliary Stricture Simulating as Cholangiocarcinoma. Journal of Clinical and Diagnostic Research: JCDR. 2015;9(3):PL01-PL02.

3. Epstein D, Mistry K, Whitelaw A, Watermeyer G, Pettengell KE.The effect of physiological concentrations of bile acids on in vitro growth of Mycobacterium tuberculosis. SAfr $M J$. 2012;102(6):522-4.

4. S Cheddie, T Bisetty, B Singh. Isolated Tuberculosis of the Gallbladder. Internet J Surgery. 2012 Volume 28 Number 2.

5. Sharma K, Sinha SK, Sharma A, et al. Multiplex PCR for Rapid Diagnosis of Gastrointestinal Tuberculosis. Journal of Global Infectious Diseases. 2013;5(2):49-53. doi:10.4103/0974-777X.112272. 